\title{
Dashboard Prototype for Improved HIV Monitoring and Reporting for Indiana
}

\author{
Yvette Temate-Tiagueu ${ }^{1}$, Kamran Ahmed ${ }^{1}$, Joseph Amlung ${ }^{2}$, Dennis Stover ${ }^{2}$, Philip J. Peters ${ }^{1}$, \\ John T. Brooks ${ }^{1}$, Sridhar Papagari Sangareddy ${ }^{1}$, Jina J. DCruz ${ }^{1}$ \\ ${ }^{1} \mathrm{CDC}$, Atlanta, Georgia, United States, ${ }^{2}$ Indiana State Department of Health, Indianapolis, Indiana, United States
}

Objective

The objective was to design and develop a dashboard prototype (DP) that integrates HIV data from disparate sources to improve monitoring and reporting of HIV care continuum metrics in Indiana. The tool aimed to support Indiana State Department of Health (ISDH) to monitor key HIV performance indicators, more fully understand populations served, more quickly identify and respond to crucial needs, and assist in planning and decision-making.

\section{Introduction}

In 2015, ISDH responded to an HIV outbreak among persons using injection drugs in Scott County [1]. Information to manage the public health response to this event and aftermath included data from multiple sources (e.g., HIV testing, surveillance, contact tracing, medical care, and HIV prevention activities). During the outbreak, access to timely and accurate data for program monitoring and reporting was difficult for health department staff. Each dataset was managed separately and tailored to the relevant HIV program area's needs. Our challenge was to create a platform that allowed separate systems to communicate with each other and design a DP that offered a consolidated view of data.

ISDH initiated efforts to integrate these HIV data sources to better track HIV prevention, diagnosis, and care metrics statewide, support decision-making and policies, and facilitate a more rapid response to future HIV-related investigations. The Centers for Disease Control and Prevention (CDC) through its Info-Aid program provided technical assistance to support ISDH's data integration process and develop a DP that could aggregate these data and improve reporting of crucial statewide metrics.

After an initial assessment phase, an in-depth analysis of requirements resulted in several design principles and lessons learned that later translated into standardization of data formats and design of the data integration process [2].

\section{Methods}

Specific design principles and prototyping methods were applied during the 9 months that lasted the DP design and development process starting from June 2017.

\section{Requirements elicitation, analysis, and validation}

The elicitation and analysis of the requirements were done using a dashboard content inventory tool to gather and analyze HIV reporting needs and dashboard requirements from stakeholders. Results of this analysis allowed us to validate project goals, list required functionalities, prioritize features, and design the initial dashboard architecture. The initial scope was Scott County.

\section{Design mapping}

The design mapping exercise reviewed different scenarios involving data visualization using DP, clarified associations among data from different programs and determined how best to capture and present them in the DP. For example, we linked data in separate datasets using unique identifier or county name. This step's output was to refine DP architecture. 


\section{Parallel design}

In a parallel design session, we drew dashboard mockups on paper with end users. These mockups helped illustrate how information captured during design mapping would be translated into visual design before prototype implementation. Drawings were converted to PowerPoint mockups for validation and modifications. The mockup helped testers and future users, interact and rapidly understand the DP architecture. The model can be used for designing other DP. Integration

Data integration was conducted in SAS by merging datasets from different program areas iteratively. Next, we cleaned (e.g., deleted records missing crucial information) and validated data. The integration step solved certain challenges with ISDH data (e.g. linking data across systems while automating data cleaning was planned for later), increased data consistency and reduced redundancy, and resulted in a consolidated view of the data.

\section{Prototyping}

After data integration, we extracted a reduced dataset to implement and test different DP features. The first prototype was in Excel. We applied a modular design that allowed frequent feedback and input from ISDH program managers. Developers of the first prototype were in two locations, but team members kept in close contact and further refined the DP through weekly communications. We expanded the DP scope from Scott County to include all counties in Indiana.

\section{Beta Version}

To enable advanced analysis and ease collaboration of the final tool across users, we moved to Tableau Desktop Professional version 10. All Excel screens were redeveloped and integrated into a unique dashboard for a consolidated view of ISDH programs. After beta version completion, usability tests were conducted to guide the DP production version.

\section{Technical requirements}

All users were provided Tableau Reader to interact with the tool. DP is not online, but shared by ISDH through a protected shared drive. Provisions are made for the DP to use a relational database that will provide greater data storage flexibility, management, and retrieval. DP benefits from the existing security infrastructure at ISDH that allows for safeguarding personal identifiable information, secured access, backup and restoration.

\section{Results System content}

ISDH's data generated at the county and state level were used to assess the following domains: HIV Testing, HIV Surveillance, Contact Tracing, HIV Care Coordination, and Syringe Exchange. The DP was populated through an offline extract of the integrated datasets. This approach sped up the Tableau workbook and allowed monthly update to the uploaded datasets. The system also included reporting features to display aggregate information for multiple population groups.

\section{Stakeholders' feedback}

To improve users' experience, the development team trained and offered stakeholders multiple opportunities to provide feedback, which was collected informally from ISDH program directors to guide DP enhancements. The initial feedback was collected through demonstration to CDC domain experts and ISDH staff. They were led through different scenarios and provided comments on overall design and suggestions for improvement. The goal of the demos was to assess ease of use and benefits and determine how it could be used to engage with stakeholders inside and outside of ISDH.

\section{DP Action Reporting}

The DP reporting function will allow users to download spreadsheets and graphs. Some reports will be automatically generated and some will be ad-hoc. All users, including the ISDH Quality Manager and grant writers, can use the tool to guide program evaluations and justifications for funding. The tool will provide a way for ISDH staff to stay current about work of grantees, document key interactions with each community, and track related next steps. In addition, through an extract of the integrated dataset (e.g., out-of-care HIV positives), DP could support another ISDH program area, Linkage to Care.

ISDS Annual Conference Proceedings 2019. This is an Open Access article distributed under the terms of the Creative Commons AttributionNoncommercial 4.0 Unported License (http://creativecommons.org/licenses/by-nc/3.0/), permitting all non-commercial use, distribution, and reproduction in any medium, provided the original work is properly cited. 


\section{Conclusions}

We describe the process to design and develop a DP to improve monitoring and reporting of statewide HIV-related data. The solution from this technical assistance project was a useful and innovative tool that allows for capture of time-crucial information about populations at high risk. The system is expected to help ISDH improves HIV surveillance and prevention in Indiana. Our approach could be adapted to similar public health areas in Indiana.

\section{References}

1. Peters PJ, et al. 2016. HIV infection linked to injection use of oxymorphone in Indiana, 2014-2015. N Engl J Med. 375(3), 229-39. PubMed https://doi.org/10.1056/NEJMoa1515195

2. Ahmed K, et al. 2018. Integrating data from disparate data systems for improved HIV reporting: Lessons learned. Online J Public Health Inform. 10(1), e49. 